\title{
Effect of Neutrophils on Gallbladder Interstitial Cajal-Like Cells in Guinea Pig Model of Acute Cholecystitis
}

\author{
Zhen-peng Huanga, ${ }^{a, b}$ Qiu ${ }^{a, b}$ Yan Yang ${ }^{a, b}$ Bao-ping Yua
}

aDepartment of Gastroenterology, Renmin Hospital of Wuhan University, Wuhan, ${ }^{\text {b}}$ Key Laboratory of Hubei Province for Digestive System Diseases, Wuhan, P.R.China

\section{Key Words}

Acute cholecystitis • Interstitial Cajal-like cells $\bullet$ c-kit • Stem cell factor

\begin{abstract}
Background: Acute cholecystitis is a common condition in gallbladder motility disorder. Interstitial Cajal-like cells (ICLCs) in the gallbladder are known as one of the players in the complex motility mechanisms affecting gallbladder motility. Aim: This study explored morphological symptoms and molecular mechanisms underlying gallbladder ICLC changes induced by acute cholecystitis. Materials and Methods: Fifteen adult guinea pigs were randomly divided into 3 groups: sham-operated group (healthy controls) and 2 experimental groups wherein these guinea pigs were subjected to common bile duct ligation to induce acute cholecystitis. Neutrophils were isolated from the peripheral blood of sham-operated animals and from the experimental animals at 24 and $48 \mathrm{~h}$ after surgery, and co-cultured with gallbladder ICLCs. The morphology of gallbladder ICLCs was examined by laser confocal immunofluorescence microscopy, TUNEL assay was used to detect apoptosis, and western blot and real-time PCR were performed to detect stem cell factor (SCF) and c-kit protein and mRNA expression, respectively. Results: No morphological differences in the gallbladder ICLCs were observed between single-culture and co-culture with healthy control neutrophil groups. However, the ICLCs in all co-culture groups with acute inflammation were impaired. In the co-culture groups, the rate of ICLC apoptosis was significantly higher than that in the singleculture group. SCF and c-kit protein and mRNA expression levels decreased in all co-culture groups as well. Conclusion: We demonstrated that the neutrophils are involved in gallbladder ICLC injury in acute cholecystitis cases and associated with gallbladder motility disorder.
\end{abstract}

\section{Introduction}

Gallbladder motility is regulated by various mechanisms, one of which is via the functioning of interstitial Cajal-like cells (ICLCs). Gallbladder ICLCs generate and spread H. Qiu and Z.-p. Huang have the same contribution in the work. 


\section{Cellular Physiology Cell Physiol Biochem 2016;39:2033-2043 \begin{tabular}{ll|l} 
DOI: 10.1159/000447899 & O 2016 The Author(s). Published by S. Karger AG, Basel \\
www.karger.com/cpb
\end{tabular} \\ Huang et al.: Neutrophils Injured Interstitial Cajal-Like Cells in Acute Cholecystitis}

spontaneous rhythmic contractions of the gallbladder, thereby promoting gastrointestinal electrical activity and mediating and regulating neurotransmitter activity in the gastrointestinal tract [1-5]. These cells have been associated with biliary disorders such as acute cholecystitis and cholelithiasis, which is a frequent reason for patients to visit to the emergency department [6-8]. However, the mechanism underlying the changes in gallbladder ICLCs in acute cholecystitis remains to be elucidated. Moreover, it is not known whether acute cholecystitis can lead to abnormalities in gallbladder ICLCs and the stem cell factor (SCF)/c-kit pathway.

This study aimed to explore the cellular and molecular mechanisms underlying the changes in gallbladder ICLCs in acute cholecystitis, using a guinea pig model in which acute cholecystitis was induced.

\section{Materials and Methods}

\section{Animals}

Fifteen 10- to 14-day-old guinea pigs (both males and females) and 15 guinea pigs (both male and female; weight 250-350 g) were obtained from Wuhan Institute of Biological Products Co., Ltd. The animals were housed under standard laboratory conditions $\left(22^{\circ} \mathrm{C} \pm 2{ }^{\circ} \mathrm{C}\right.$ with a 12 -h light/dark cycle and a relative humidity of 40-60\%), and were given access to diet and water ad libitum. All animal experiments were approved by the Institutional Animal Care and Use Committee of Wuhan University, and the study protocol was designed to minimize pain and discomfort to the animals.

The 250-350 g guinea pigs were randomly divided into 3 groups (5 animals per group). The shamoperated group comprised healthy control animals. The animals in the two study groups underwent common bile duct ligation (CBDL), in order to induce acute cholecystitis. Further, $1 \mathrm{ml}$ of peripheral blood was collected by syringes from the animals in the 24-h and 48-h CBDL groups, for the isolation and quantification of neutrophils at 24 and $48 \mathrm{~h}$ post-surgery, respectively. Afterward, these animals were subjected to laparotomy and cholecystectomy as previously described [9-11].

Hematoxylin-eosin staining was performed for all gallbladder specimens from each group to detect the degree of acute cholecystitis using light microscopy (Olympus BX53; Olympus, Tokyo, Japan).

\section{Isolation of Neutrophils}

Peripheral blood neutrophils from guinea pigs were separated using a peripheral blood lymphocyte separation medium (TBD, Tianjing, China), according to the manufacturer's instructions.

\section{Gallbladder ICLC Culture}

For gallbladder ICLC culture, 10- to 14-day-old guinea pigs were sacrificed by cervical dislocation and subjected to laparotomy and cholecystectomy. Gallbladders were removed with an intact mesenteric vascular bed to minimize stretch when transferred to a dissection dish containing D-Hank's solution. The gallbladder specimens were cut and whole-mounts of the muscular layer were prepared, whereas the mucosal and submucosal layers were removed under a surgical microscope. The tissue specimens were transferred to a dissection dish containing fetal bovine serum (FBS; Gibco, Australia), and cut into muscle strips (1-2 mm in diameter). These muscle strips were transferred into a cell culture flask (Sigma, St. Louis, USA) with M199 medium (Hyclone, Beijing, China) containing $20 \% \mathrm{FBS}$ and incubated at $37^{\circ} \mathrm{C}$ for 4 days, under $95 \% \mathrm{O}_{2}$ and $5 \% \mathrm{CO}_{2}$ conditions before co-culturing $[12,13]$.

\section{Co-culture of Gallbladder ICLCs and Neutrophils}

Neutrophils isolated from the $1 \mathrm{ml}$ peripheral blood samples of 250-350g guinea pigs from the sham-operated group (co-culture 1 group), 24-h CBDL group (co-culture 2 group), and 48-h CBDL group (co-culture 3 group) were cultured with gallbladder ICLCs in a cell culture flask (Sigma, St. Louis, USA) containing RPMI-1640 medium (Hyclone, Beijing, China) supplemented with $10 \% \mathrm{FBS}$, at $37^{\circ} \mathrm{C}$ for $24 \mathrm{~h}$, in $95 \% \mathrm{O}_{2}$ and $5 \% \mathrm{CO}_{2}$, followed by the removal of the neutrophils from the cell culture flasks. 


\section{Cellular Physiology Cell Physiol Biochem 2016;39:2033-2043 \begin{tabular}{l|l|l|} 
and BOI: 10.1159/000447899 & $\begin{array}{l}\text { C) 2016 The Author(s). Published by S. Karger AG, Basel } \\
\text { www.karger.com/cpb }\end{array}$
\end{tabular} \\ Huang et al.: Neutrophils Injured Interstitial Cajal-Like Cells in Acute Cholecystitis}

Immunohistofluorescence and Identification of ICLCS

Cultured ICLCs stained on the slides and fixed in $4 \%$ polyformaldehyde solution and stored at $4{ }^{\circ} \mathrm{C}$ for $30 \mathrm{~min}$. They were subsequently washed thrice with phosphate-buffered saline (PBS) for $10 \mathrm{~min}$. Afterward, the samples were immersed in PBS with $0.3 \%$ Triton X-100, followed by blocking with normal goat serum for $30 \mathrm{~min}$ at $20^{\circ} \mathrm{C}$. Next, the samples were incubated with primary rat monoclonal anti-c-kit antibody (concentration was 1:50, eBioscience, San Diego, USA) overnight at $4^{\circ} \mathrm{C}$ and kept the slides in the dark. Then, the gallbladder cells were washed thrice, for $10 \mathrm{~min}$ each, with Tris-buffered saline and Tween-20 solution (TBST) and incubated with CY3-conjugated rabbit anti-rat secondary antibody(concentration was 1:100, Boster, Wuhan, China) in PBS for $1 \mathrm{~h}$ at room temperature. After removing the unbound secondary antibody by washing with TBST for $5 \mathrm{~min}$, three times, images were captured using a laser confocal microscope (Olympus FV1000, Tokyo, Japan).

\section{Detection of ICLC Apoptosis}

TUNEL (In Situ Cell Death Detection Kit, Fluorescein, Roche Applied Science, Mannheim, Germany) assay was used to detect gallbladder ICLCs death in the single-culture group and three co-culture groups, following the manufacturer's instructions. Images were captured by a laser confocal microscope (Olympus FV1000; Tokyo, Japan).

Protein Extraction and Western Blot Analysis

Total proteins from all co-culture and ICLC groups were extracted using RIPA lysis buffer (Beyotime, Shanghai, China) and subsequently subjecting to centrifugation at $12000 \mathrm{~g}$, at $4^{\circ} \mathrm{C}$ for $5 \mathrm{~min}$. Afterward, supernatants were collected and protein concentration was estimated using the BCA protein assay kit (Beyotime, Shanghai, China). Thirty micrograms of total proteins were loaded on $10 \%$ SDS polyacrylamide gels. The separated proteins were transferred to a nitrocellulose membrane (Pierce Biotechnology, Rockford, USA), and the membranes were incubated with $5 \%$ skimmed milk at room temperature for $2 \mathrm{~h}$, to block nonspecific binding sites. The samples were incubated with the primary antibody against c-kit (eBioscience, San Diego, USA) or SCF (Abcam, Cambridge, UK) overnight at $4^{\circ} \mathrm{C}$. After washing three times with TBST for 10 min, the secondary antibody of the matching conjugated horseradish peroxidase was applied for $1 \mathrm{~h}$ at room temperature, followed three washes with TBST for $10 \mathrm{~min}$. Specific protein bands were visualized using an X-ray film (Kodak, Xiamen, China) and the chemiluminescence detection kit (Amersham, Pittsburgh, USA). Optical density of the bands was analyzed using Band Scan 5.0 software (Alpha Innotech Corp., California, USA).

\section{RNA Extraction}

Total RNA was extracted from all co-cultured ICLCs and single cultured-ICLCs wtih TRIzol reagent (Invitrogen, Carlsbad, USA), following the manufacturer's instructions.

\section{RT-PCR Analysis}

cDNA was reverse-transcribed using $1.13 \mu \mathrm{g}$ of total RNA and amplified by 40 cycles of denaturation $\left(2 \mathrm{~min}\right.$ at $50^{\circ} \mathrm{C}, 10 \mathrm{~min}$ at $\left.95^{\circ} \mathrm{C}\right)$, annealing $\left(30 \mathrm{~s}\right.$ at $\left.95^{\circ} \mathrm{C}\right)$ and synthesis ( $30 \mathrm{~s}$ at $\left.60^{\circ} \mathrm{C}\right)$. GAPDH primers were as follows: 5'-ATCACTGCCACCCAGAAGACT-3' (forward) and 5'-CAGATCCACAACCGACACATTA-3' (reverse), generating $195 \mathrm{bp}$ long amplicons. SCF primers were 5'-GAAAGATTCCAGAGTCAGTGTCA-3' (forward) and 5'-AAGCAAAGCCAATCACAAGAG-3' (reverse), and the amplicons generated were $179 \mathrm{bp}$ long. The primers for c-kit were 5'-TATCCTCCTTACTCATGGTCGAA-3' (forward) and 5'-CGGGCATTTCCTTTAACCACATA-3' (reverse), and the amplicons generated were $99 \mathrm{bp}$ long. Afterward, PCR products were separated by electrophoresis on a $1.5 \%$ agarose gel stained with ethidium bromide. GAPDH levels were used as an internal reference control, and mRNA levels were expressed as relative units (Table 1).

\section{Statistical Analysis}

All statistical analyses were performed using SPSS for Windows version 17.0 (SPSS, Chicago, USA). Continuous variables were compared using the $t$-test and are presented as mean \pm standard deviation (SD). Categorical variables were compared using ANOVA. A two-sided $P$ value $<0.05$ was regarded as statistically significant. 


\section{Cellular Physiology Cell Physiol Biochem 2016;39:2033-2043

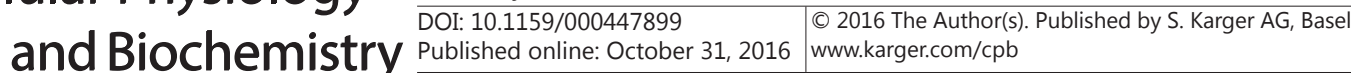

Table 1. Study Methods Flow Chart

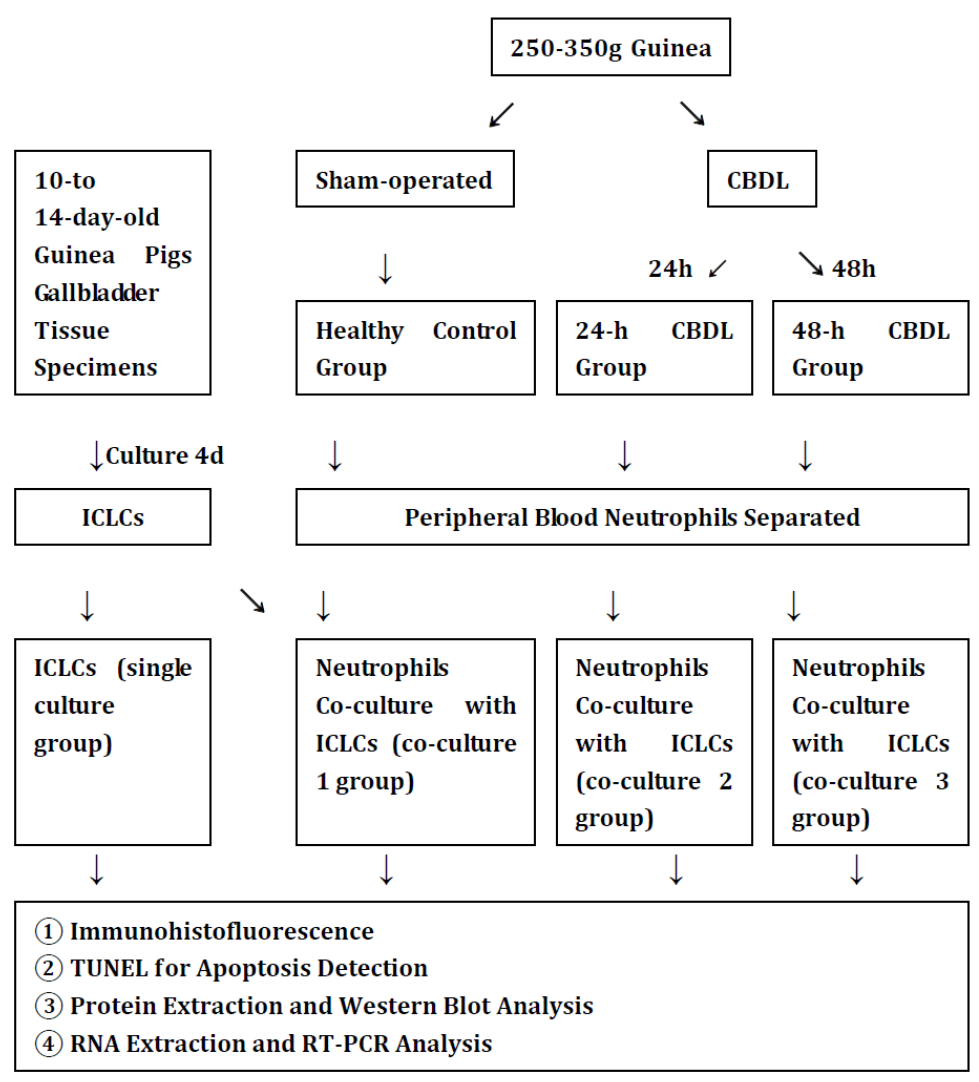

\section{Results}

\section{Evaluation of Animal Model}

To ensure that animals survive, guinea pigs were kept in the quiet environment and given access to diet and water after surgery. No guinea pigs from the sham-operated group, and the 24-h and 48-h CBDL groups died during the experiments.

Histopathological analyses revealed that the gallbladder tissue obtained from the shamoperated animals was intact and without obvious inflammatory cell infiltration. However, gallbladder tissue isolated from the 24-h CBDL group showed edema, hyperemia, and considerable inflammatory cell infiltration. Gallbladder tissue from the 48-h CBDL group showed edema, hyperplasia of fibroblasts, hyperemia, hemorrhage, and considerable inflammatory cell infiltration (mainly neutrophils) in the muscularis mucosae (Fig. 1), showing that acute cholecystitis was induced in CBLD guinea pigs.

\section{Immunohistofluorescence Analysis and Identification of ICLCS}

Immunohistofluorescence analysis revealed that gallbladder ICLCs mainly had an astrocyte-like or fusiform shape, and a diameter of about 7-10 $\mu \mathrm{m}$. ICLCs that stained positive for c-kit had large, orbicular, or ovate nuclei, reduced cytoplasm, and scattered chromatin. Each ICLC was shown to have 2-5 synapses. These cells typically appeared as single cells or in small clusters of 2-3 cells, connected to each other, forming a network-like structure. Additionally, mast cells stained positively for c-kit, and were round. However, mast cells were only occasionally observed with very short processes (Fig. 2).

No significant morphological differences were observed between gallbladder ICLCs cultured alone or in co-culture 1 group. In contrast to this, in both co-culture 2 group and coculture 3 group, gallbladder ICLCs synapses was shortened, with fewer connections between ICLCs, and the ICLC network structure was partly broken. Furthermore, fewer or no mast 


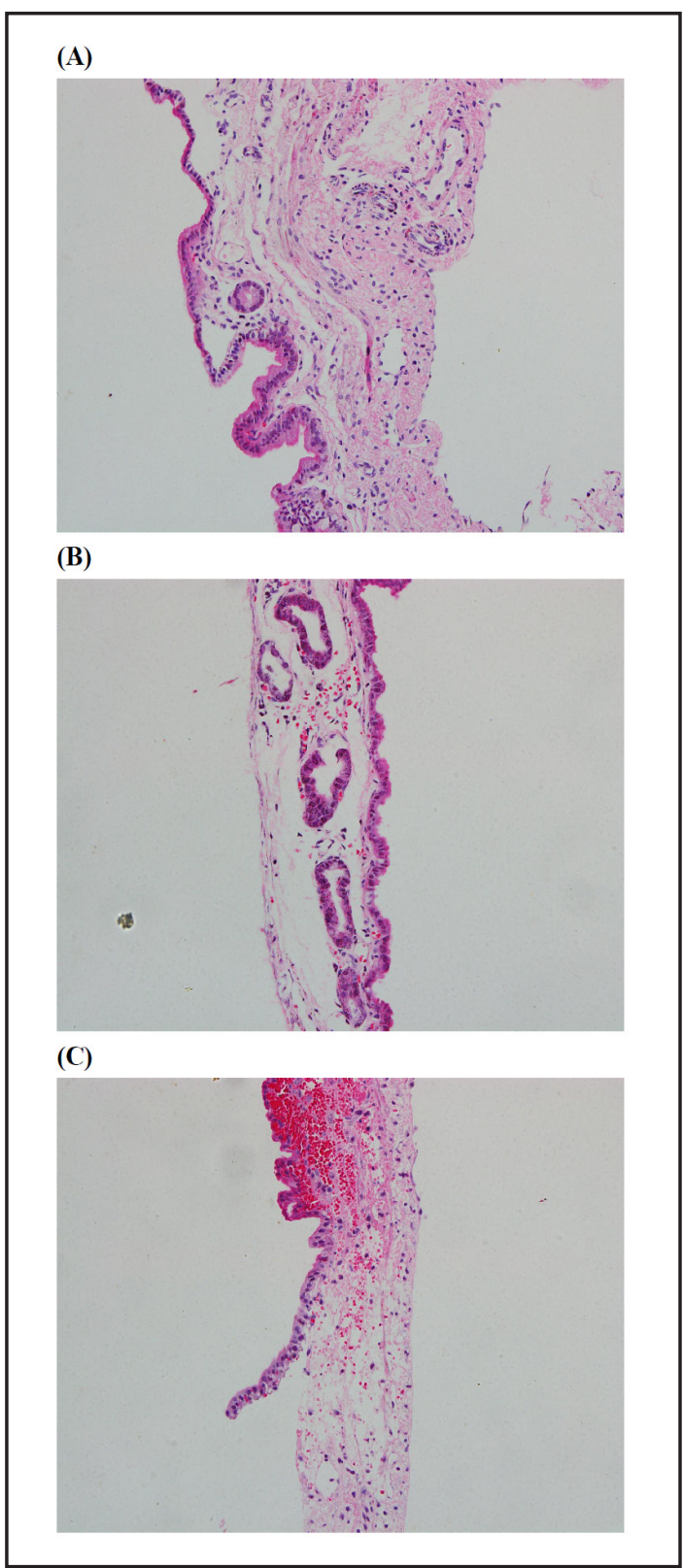

Fig. 1. Histopathological analysis of Animal Model. (A) Gallbladder tissue from the sham-operated group was found to be intact and without obvious inflammatory cell infiltration. (B) Gallbladder tissue from the 24-h CBDL group showed edema, blood-expanded hyperemia, and considerable inflammatory cell infiltration. (C) Gallbladder tissue from the 48-h CBDL group showed edema, hyperplasia of fibroblasts, blood-expanded hyperemia, hemorrhage, and considerable inflammatory cell infiltration (mainly was neutrophils) in the mucosa and muscularis.

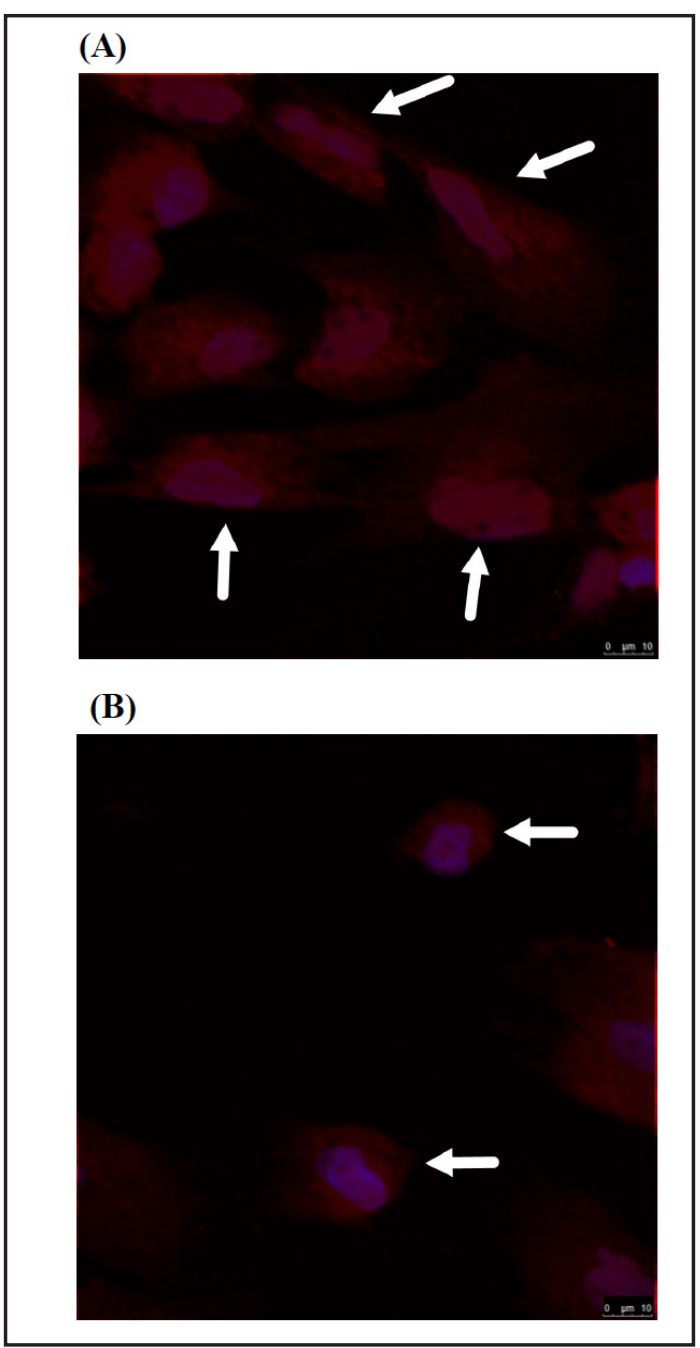

Fig. 2. Identification of ICLCs. Cultured ICLCs stained on the slides and examined by laser confocal immunofluorescence microscopy. (A) Gallbladder ICLCs mainly had an astrocyte or fusiformis shape, and stained positive for c-kit had large, orbicular, or ovate nuclei (blue); reduced cytoplasm (red); and scattered chromatin. Each ICLC also had 2-5 long synapses. These cells typically appeared singly or in small clusters of two or three cells and were connected with each other to form a network-like structure. (B) Mast cells were also immunolabeled positively for Kit, and were observed as round cell bodies (white arrows).

cells were observed. The morphological changes were more pronounced in co-culture 3 group than in co-culture 2 group (Fig. 3).

\section{KARGER}




\section{Cellular Physiology and Biochemistry

Fig. 3. Immunohistofluorescence Analysis. Single-culture group ICLCs and co-culture groups ICLCs all stained on the slides and examined by laser confocal immunofluorescence microscopy. Gallbladder ICLCs (white arrows) did not reveal significant morphological differences between the single-culture group (A) and the co-culture 1 group (B). In contrast, in both the co-culture 2 group (C) and the co-culture 3 group (D), gallbladder ICLCs synapses was shortened, with fewer connections among ICLCs, and the ICLC network structure was partly broken. In addition, fewer to no mast cells were observed. The morphological changes were more pronounced in co-culture 3 group than in co-culture 2 group. Mast cells were indicated with red arrows.

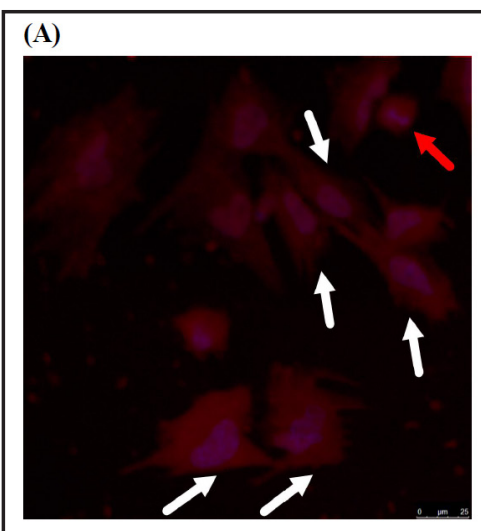

(C)

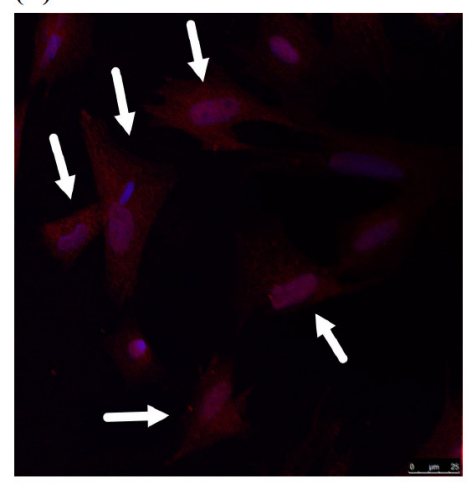

(B)

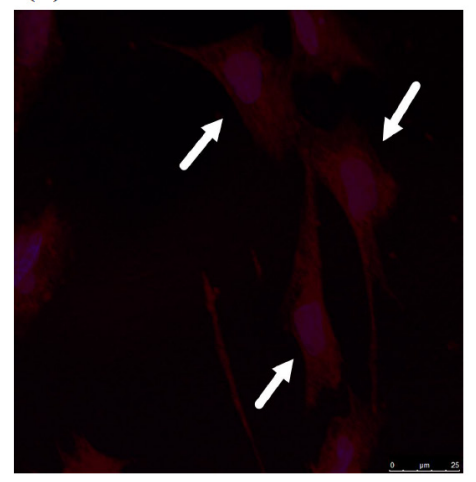

(D)

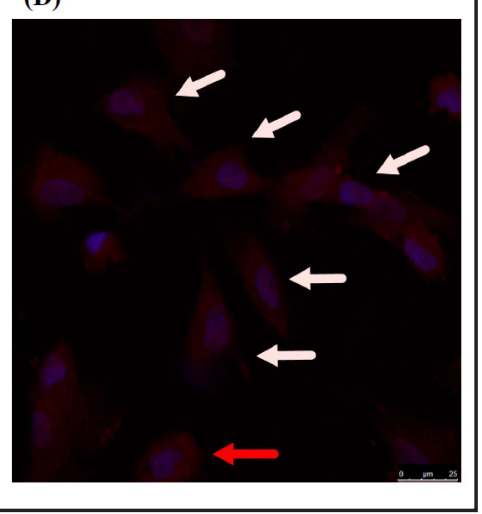

TUNEL Assay

The number of apoptotic ICLCs was significantly higher in the co-culture groups than when cultured alone, with the number being higher in co-culture 1 group than when ICLCs were cultured alone, and co-culture 3 group showing a higher number of apoptotic cells than co-culture 2 group (the number of apoptotic ICLCs in the single culture group, co-culture 1 group, co-culture 2 group, and co-culture 3 group were $1.0000 \pm 0.00000,1.6667 \pm 0.57735$, $5.3333 \pm 0.57735,10.6667 \pm 0.57735, F=192.889, P=0.000$ ) (Fig. 4).

\section{SCF and c-kit Protein Expression in ICLCs}

Western blot analysis revealed that the protein levels of both c-kit and SCF are considerably lower in the co-culture groups than in the single culture group, and the lowest levels of both were detected in co-culture 3 group. The obtained SCF and $\beta$-actin level ratios in the single culture group, co-culture 1 group, co-culture 2 group, and co-culture 3 group were $0.4553 \pm 0.04717,0.4323 \pm 0.05613,0.2790 \pm 0.04107$, and $0.1487 \pm 0.01620$, respectively $(F=33.670, P=0.000)$, and c-kit and $\beta$-actin ratios were $0.5943 \pm 0.07095$, $0.5503 \pm 0.03109,0.3363 \pm 0.04712$, and $0.2327 \pm 0.04974$, respectively $(F=33.360, P=$ 0.000) (Fig. 5), showing that SCF and c-kit protein expression was decreased in all co-culture groups.

\section{SCF and c-kit mRNA Expression in ICLCs}

RT-PCR analysis revealed that the SCF and c-kit mRNA levels decreased in all three co-culture groups, and they were shown to be the lowest in co-culture 3 group. Compared with the single-culture group, in co-culture 1 group, co-culture 2 group, and co-culture 3 group, SCF mRNA levels were $1.0000 \pm 0.00000$ vs. $0.9673 \pm 0.02887$ vs. $0.6630 \pm 0.07119$ vs. $0.4350 \pm 0.14584$, respectively, and c-kit mRNA levels were $1.0000 \pm 0.0000$ vs. $0.9570 \pm$ 0.02961 vs. $0.7130 \pm 0.05839$ vs. $0.4287 \pm 0.10130$, respectively $(F=31.721$ and $F=57.066$, respectively; all $P=0.000$ ) (Fig. 6). 
Fig. 4. TUNEL Assay. Single-culture group ICLCs and co-culture groups ICLCs all stained on the slides and examined by TUNEL Assay. The density of ICLCs that underwent apoptosis was significantly higher in the co-culture groups than in the single-culture group (A), with co-culture 1 group (B) showing a higher density than the single-culture group, and co-culture 3 group (D) showing a higher density than co-culture 2 group(C), too(1.0000 \pm 0.00000 vs. $1.6667 \pm 0.57735$ vs. $5.3333 \pm 0.57735$ vs. $10.6667 \pm$ $0.57735, F=192.889, P=0.000$ ).

\section{Discussion}

ICLCs were first described in the gastrointestinal tract by Santiago Ramón y Cajal in $1893[14,15]$. In the last century, several studies have found that ICLCs exist in the gut of both human beings and animals, and they have been detected in the esophagus, stomach, small intestine, large bowel, ileum, and colon [16-18]. More recently, studies have found that ICLCs are distributed in the gallbladder and extrahepatic biliary duct of both guinea-pigs and humans [18-21]. Gallbladder motility is regulated by various mechanisms, for instance, the activity of the gallbladder smooth muscle and the nervous circuit, which includes gallbladder ICLCs [15]. The morphology of ICLCs observed here was consistent with that observed in a previous report [6].

ICLCs in the biliary system have been associated with various biliary systemic diseases, such as acu-

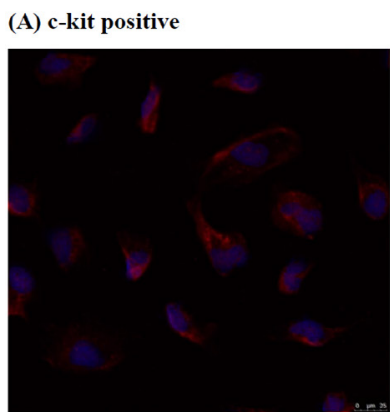

(B) c-kit postive

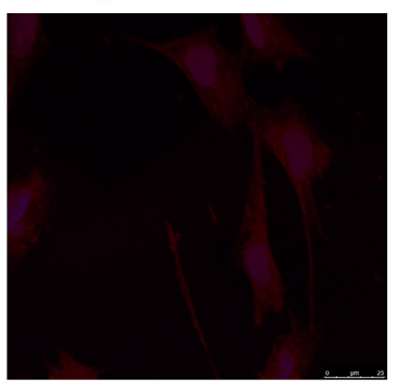

(C) c-kit postive

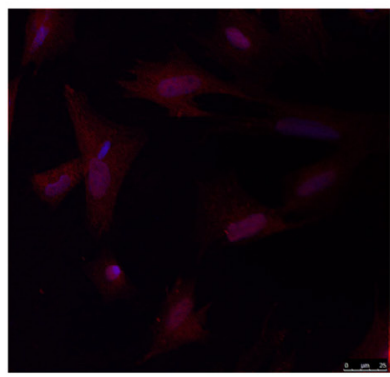

(I) c-kit postive

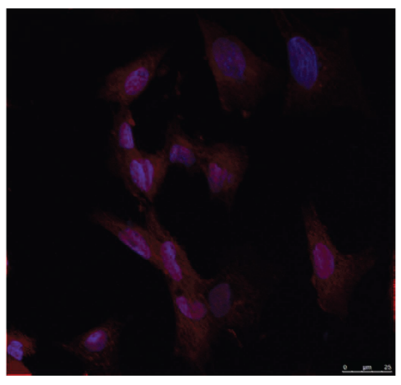

(E)

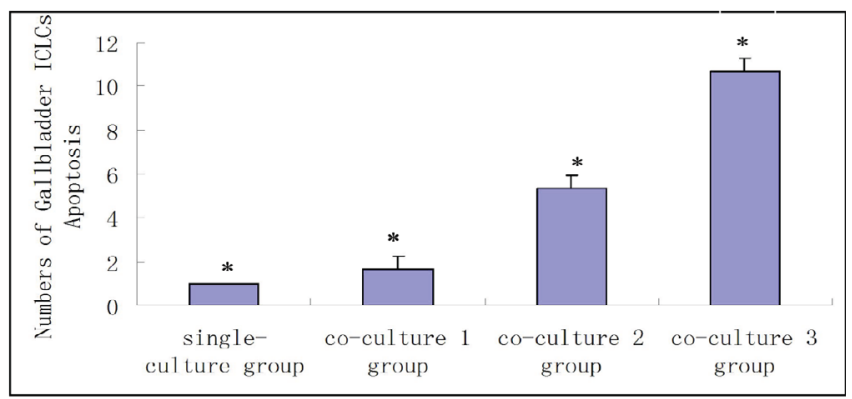

* $F=192.889, P=0.000$

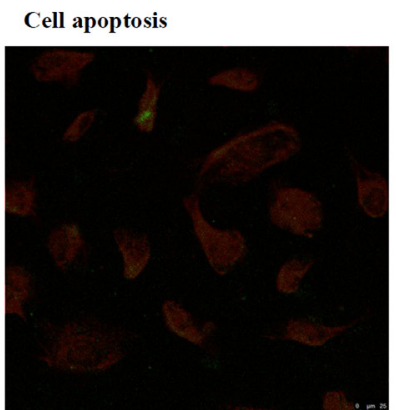

Cell apoptosis

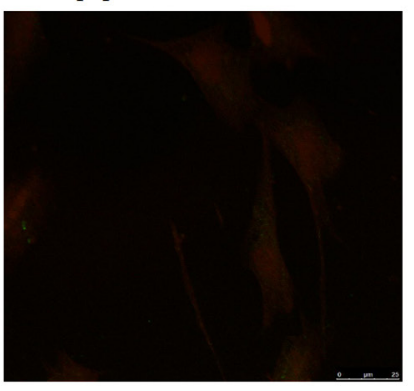

Cell apoptosis

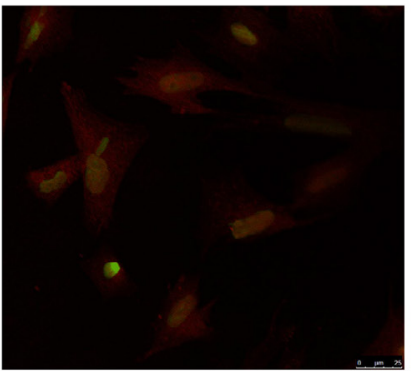

Cell apoptosis

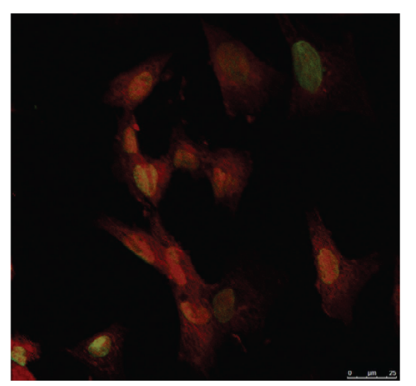




\section{Cellular Physiology Cell Physiol Biochem 2016;39:2033-2043

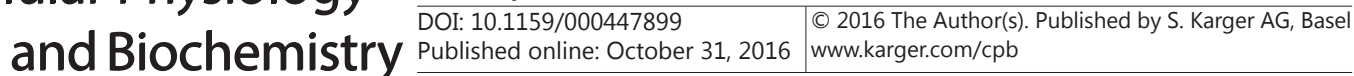

Fig. 5. SCF and c-kit protein expression in different groups. The protein levels of both c-kit (A) and SCF (B) were significantly lower in the co-culture groups (lanes $2,3,4$ ) than in the single culture group (lane 1). Both SCF and c-kit protein levels were the lowest in co-culture 3 group. The ratios of SCF and $\beta$-actin (C) in the single culture group, co-culture 1 group, co-culture 2 group, and co-culture 3 group were $0.4553 \pm 0.04717,0.4323 \pm$ $0.05613,0.2790 \pm 0.04107$, and $0.1487 \pm 0.01620$, respectively $(F=33.670, P=$ 0.000 ), and the ratios of c-kit and $\beta$-actin were $0.5943 \pm$ 0.07095, $0.5503 \pm 0.03109$, $0.3363 \pm 0.04712$, and $0.2327 \pm 0.04974$, respectively $(F=33.360, P=0.000)$.

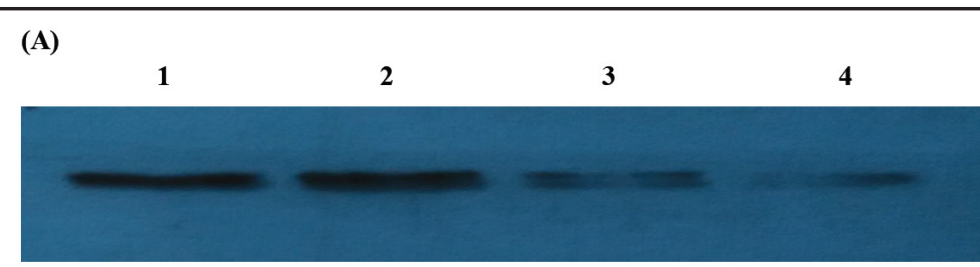

(B)
1 2 3 4

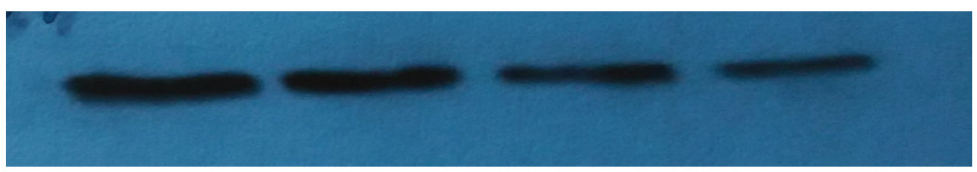

(C)
2

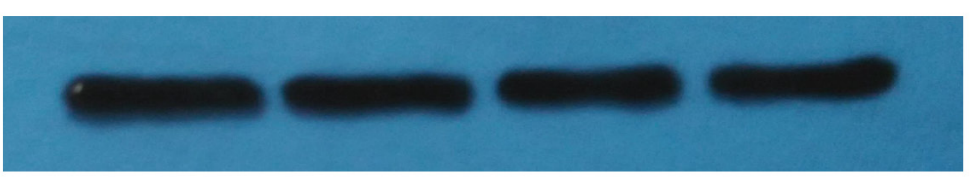

(D)

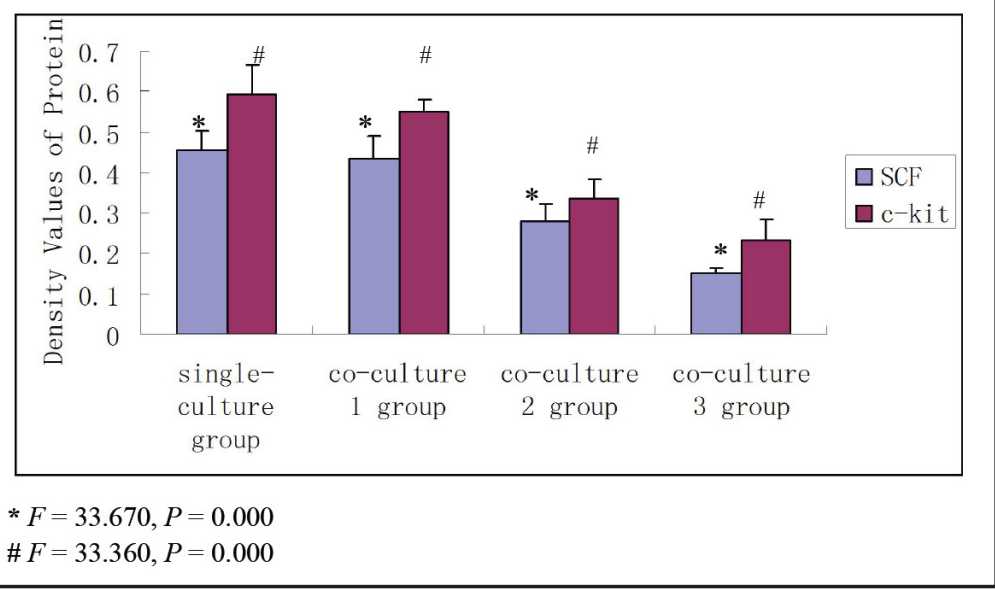

te inflammation, regional obstruction, and subsequent proximal dilation and injury to the nervous and immune systems, and ICLCs damage and/or loss has been observed in almost every gastrointestinal motility disorder, with abnormalities in motor activity impairing regional transit and symptoms [22, 23]. Previous studies have also shown that ICLCs undergo apoptosis over time and that their regeneration is important for the maintenance of normal ICLC networks [24]. In this study, when co-cultured with the inflammatory cells, gallbladder ICLCs underwent morphological changes, and a lower number of mast cells were observed. The morphological changes were more pronounced within more acute inflammation processes. Additionally, consistent with the results obtained in a previous study, mast cells stained positively for c-kit, and were observed to be round in vivo and in vitro, with small processes [19]. The results of the TUNEL assay strongly indicate that neutrophils obtained from the guinea pigs with acute cholecystitis may increase the apoptosis of gallbladder ICLCs. The increased number of apoptotic ICLCs may lead to impaired regeneration and maintenance of the ICLC network. Increased gallbladder ICLC apoptosis and the disruption of ICLC network may have a substantial effect on ICLCs function, and may lead to gallbladder motility disorder.

Gallbladder ICLCs act as the initiators of pacemaker activity and modulating gallbladder motility, and mediate enteric motor neurotransmission [25]. Pervious studies have showed that capacitive-resistive electric transfer, TRPM7/ANO1 and LPA1/3 signaling pathways 


\section{Cellular Physiology Cell Physiol Biochem 2016;39:2033-2043 and BiOChemistry Published online: October 31, 2016 le $\begin{aligned} & \text { DOI: } 2016 \text { The Author(s). Published by S. Karger AG, Base } \\ & \text { www.karger.com/cpb }\end{aligned}$
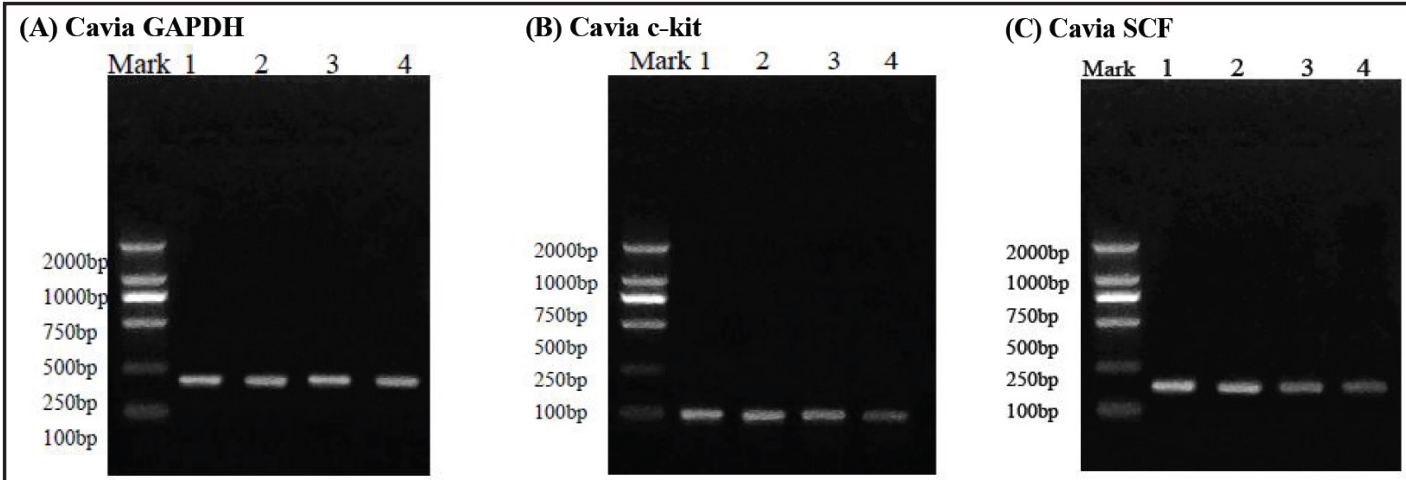

(D)

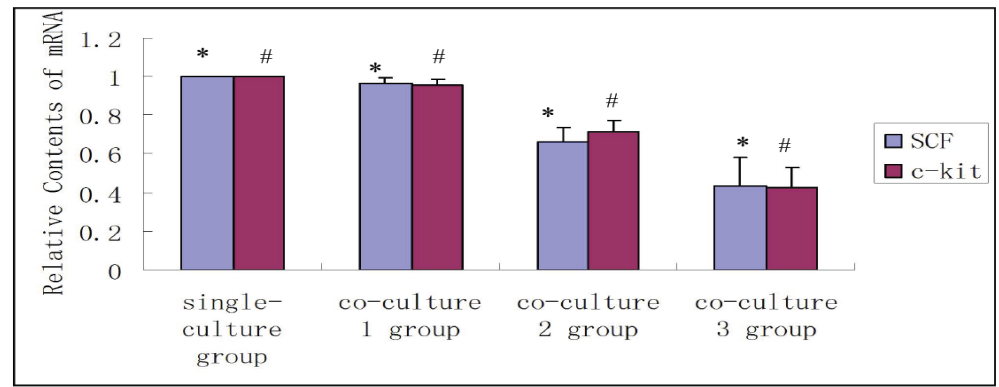

$* F=31.721, P=0.000$

$\# F=57.066, P=0.000$

Fig. 6. mRNA expression of SCF and c-kit in different groups. SCF and c-kit mRNA levels were much lower in co-culture 3 group than in the other groups. Compared with the single-culture group (lane 1), in co-culture 1 group (lane 2, co-culture 2 group (lane 3), and co-culture 3 group (lane 4), SCF mRNA levels were $1.0000 \pm$ 0.00000 vs. $0.9673 \pm 0.02887$ vs. $0.6630 \pm 0.07119$ vs. $0.4350 \pm 0.14584$, respectively, and c-kit mRNA levels were $1.0000 \pm 0.0000$ vs. $0.9570 \pm 0.02961$ vs. $0.7130 \pm 0.05839 v s$. $0.4287 \pm 0.10130$, respectively ( $F$-values were 31.721 and 57.066 , respectively; all $P=0.000$ ).

may have an effect on ICLCs [26-28]. Recent studies have demonstrated that tyrosine kinase receptor c-kit and its ligand SCF play an important role in the normal development, maturation, and phenotype maintenance of ICLCs $[29,30]$, and that c-kit represents a specific marker expressed by ICLCs [19]. When this receptor is blocked, ICLC survival is greatly affected [29]. However, co-culture of ICLCs with neutrophils derived from the peripheral blood of animal models of acute inflammation was shown to induce the injury of gallbladder ICCs, block c-kit and SCF expression, and subsequently suppress SCF/c-kit pathway activation [31]. However, the mechanism of SCF/c-kit pathway suppression remains unknown. Here, we observed that SCF/c-kit protein and mRNA expression levels decreased when ICLCs were co-cultured with neutrophils derived from the peripheral blood of acute cholecystitis-induced animals.

The obtained results suggest that neutrophils in animals with acute inflammation affect the development and function of gallbladder ICLCs, through the regulation of SCF/c-kit expression. However, the detailed mechanisms underlying this process need to be further elucidated. Additionally, this inflammation-induced ICLC injury is associated with the changes in gallbladder motility in acute cholecystitis.

\section{Acknowledgement}

This study was supported by National Natural Science Foundation of China (NSFC) (NO. 81170351). 


\title{
Cellular Physiology Cell Physiol Biochem 2016;39:2033-2043

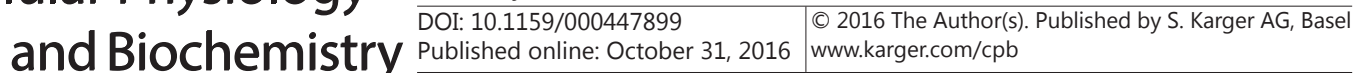 \\ Huang et al.: Neutrophils Injured Interstitial Cajal-Like Cells in Acute Cholecystitis
}

\section{Disclosure Statement}

\author{
No conflict of interest.
}

\section{References}

1 Sanders KM, Ward SM, Koh SD: Interstitial cells: regulators of smooth muscle function. Physiol Rev 2014;94:859-907.

2 Abramovic M, Radenkovic G, Velickov A: Appearance of interstitial cells of Cajal in the human midgut. Cell Tissue Res 2014;356:9-14.

3 Lies B, Gil V, Groneberg D, Seidler B, Saur D, Wischmeyer E, Jiménez M, Friebe A: Interstitial cells of Cajal mediate nitrergic inhibitory neurotransmission in the murine gastrointestinal tract. Am J Physiol Gastrointest Liver Physiol 2014;307:G98-G106.

4 Huizinga JD, Chen JH, Mikkelsen HB: Interstitial cells of Cajal, from structure to function. Front Neurosci 2013;7:43.

5 Huizinga JD, Chen JH: Interstitial cells of Cajal: update on basic and clinical science. Curr Gastroenterol Rep 2014;16:363.

6 Pasternak A, Gil K, Matyja A, Gajda M, Sztefko K, Walocha JA, Kulig J, Thor P: Loss of gallbladder interstitial Cajal-like cells in patients with cholelithiasis. Neurogastroenterol Motil 2013;25:e17-e24.

7 Gevaert T, Lerut E, Joniau S, Franken J, Roskams T, De Ridder D: Characterization of subepithelial interstitial cells in normal and pathological human prostate. Histopathology 2014;65:418-428.

8 Ambe PC, Papadakis M, Zirngibl H: A proposal for a preoperative clinical scoring system for acute cholecystitis. J Surg Res 2016;200:473-479.

9 Zhang L, Pan C, Yang B, Xiao Y, Yu B: Enhanced expression of cystathionine $\beta$-synthase and cystathionine $\gamma$-lyase during acute cholecystitis-induced gallbladder inflammation. PLoS One 2013;8:e82711.

10 Tag CG, Weiskirchen S, Hittatiya K, Tacke F, Tolba RH, Weiskirchen R: Induction of experimental obstructive cholestasis in mice. Lab Anim 2015;49:70-80.

11 Soylu S, Aydin C, Bagcivan I, Yildirim S, Koyuncu A, Topcu O, Arici S: Effects of NO/L-arginine pathway on gallbladder contractility in bile duct ligated guinea pigs. J Surg Res 2009;155:70-76.

12 Jing Ye, Yaohui Zhu, Waliul I, Yildirim S, Koyuncu A, Topcu O, Arici S: IL-9 enhances growth of ICC, maintains network structure and strengthens rhythmicity of contraction in culture. J Cell Mol Med 2006;10:687-694.

13 Bin Zhao, Wei Liu, Rongde Wu: Co-culture of neuroepithelial stem cells with interstitial cells of Cajal results in neuron differentiation. Int J Clin Exp Med 2015;8:10437-10443.

14 Sanders KM, Ward SM: Interstitial cells of Cajal: a new perspective on smooth muscle function. J Physiol 2006;576:721-726.

15 Thuneberg L: One hundred years of interstitial cells of Cajal. Microsc Res Tech 1999;47:223-238.

16 Tang D, Xiao L, Han J, Tang D, Xiao L, Han J, Yu B, Zhang W, Mei F: Late embryonic and postnatal development of interstitial cells of cajal in mouse esophagus: distribution, proliferation and kit dependence. Cells Tissues Organs 2012;196:175-188.

17 Zheng H, Park KS, Koh SD, Tang D, Xiao L, Han J, Yu B, Zhang W, Mei F: Expression and function of a T-type Ca2+ conductance in interstitial cells of Cajal of the murine small intestine. Am J Physiol Cell Physiol 2014;306:C705-C713.

18 Jiao HY, Kim DH, Ki JS, Ryu KH, Choi S, Jun JY: Effects of lubiprostone on pacemaker activity of interstitial cells of cajal from the mouse colon. Korean J Physiol Pharmacol 2014;18:341-346.

19 Huang Y, Mei F, Yu B, Zhang HJ, Han J, Jiang ZY, Zhou DS: Distribution of the interstitial Cajal-like cells in the gallbladder and extrahepatic biliary duct of the guinea-pig. Acta Histochem 2009;111:157-165.

20 Hinescu ME, Ardeleanu C, Gherghiceanu M, Zhang HJ, Han J, Jiang ZY, Zhou DS: Interstitial Cajal-like cells in human gallbladder. J Mol Histol 2007;38:275-284.

21 Ahmadi O, Nicholson Mde L, Gould ML, Mitchell A, Stringer MD: Interstitial cells of Cajal are present in human extrahepatic bile ducts. J Gastroenterol Hepatol 2010;25:277-285.

22 Jan D, Huizinga, Natalia Zarate, Gianrico Farrugia: Physiology, injury and recovery of interstitial cells of Cajal: basic and clinical science. Gastroenterology 2009;137:1548-1556. 


\section{Cellular Physiology Cell Physiol Biochem 2016;39:2033-2043 \begin{tabular}{ll|l} 
DOI: 10.1159/000447899 & $\begin{array}{l}\text { O 2016 The Author(s). Published by S. Karger AG, Basel } \\
\text { www.karger.com/cpb }\end{array}$
\end{tabular} \\ Huang et al.: Neutrophils Injured Interstitial Cajal-Like Cells in Acute Cholecystitis}

23 Torihashi S, Nishi K, Tokutomi Y, Nishi T, Ward S, Sanders KM: Blockade of kit signaling induces transdifferentiation of interstitial cells of cajal to a smooth muscle phenotype. Gastroenterology 1999;117:140-148.

24 Simon J Gibbons, Roberto De Giorgio, Maria Simonetta Faussone Pellegrini, Garrity-Park MM, Miller SM, Schmalz PF, Young-Fadok TM, Larson DW, Dozois EJ, Camilleri M,Stanghellini V, Szurszewski JH, Farrugia G: Apoptotic Cell Death of Human Interstitial Cells of Cajal. Neurogastroenterol Motil 2009;21:85-93.

25 Fan Y, Wu S, Fu B, Weng C, Wang X: The role of interstitial Cajal-like cells in the formation of cholesterol stones in guinea pig gallbladder. Hepatol Int 2015;9:612-620.

26 María Luisa Hernández-Bule, Carlos Luis Paíno María Ángeles Trillo, Alejandro Úbeda: Electric Stimulation at $448 \mathrm{kHz}$ Promotes Proliferation of Human Mesenchymal Stem Cells. Cellular Physiology and Biochemistry 2014;34:1741-1755.

27 Byung Joo Kim, Joo Hyun Nam, Kyun Ha Kim, Myungsoo Joo, Tal Soo Ha, Kwon Yeon Weon, Seok Choi, Jae Yeoul Jun, Eun Jung Park, Jinhong Wie, Insuk So, Seung-Yeol Nah: Characteristics of Gintonin-Mediated Membrane Depolarization of Pacemaker Activity in Cultured Interstitial Cells of Cajal. Cellular Physiology and Biochemistry 2014;34:873-890.

28 Huijin Gim, Joo Hyun Nam, Soojin Lee, Ji Hwan Shim, Hyun Jung Kim, Ki-Tae Ha, Byung Joo Kim: Quercetin Inhibits Pacemaker Potentials via Nitric Oxide/cGMP-Dependent Activation and TRPM7/AN01 Channels in Cultured Interstitial Cells of Cajal from Mouse Small Intestine. Cellular Physiology and Biochemistry 2015;35:2422-2436.

29 Sanders KM, Ordög T, Koh SD, Torihashi S, Ward SM: Development and plasticity of interstitial cells of Cajal. Neurogastroenterol Motil 1999;11:311-338.

30 Fan Y, Wu S, Fu B, Yan X, Wang X, Zhang W: Decreased expression of stem cell factor mRNA and protein in the gallbladders of guinea pigs fed on high cholesterol diet. Int J Clin Exp Med 2015;8:6379-6383.

31 Rich A, Miller SM, Gibbons SJ, Malysz J, Szurszewski JH, Farrugia G: Local presentation of Steel factor increases expression of c-kit immunoreactive interstitial cells of Cajal in culture. Am J Physiol Gastrointest Liver Physiol 2003;284:G313-G320. 